\title{
Antena de Microfita de Baixo Custo para GPS
}

\author{
D.C. Nascimento, R. Schildberg, J.C. da S. Lacava e L. Cividanes
}

\begin{abstract}
Resumo-Neste trabalho é proposta uma nova metodologia para o projeto de antenas de microfita de baixo custo para aplicação em GPS. Resultados são apresentados e discutidos.
\end{abstract}

Palavras-Chave-Antenas e microfita, polarização circular, substrato de baixo custo, GPS.

Abstract-This work describes a new methodology to design lowcost circularly polarized microstrip antenna for GPS application. Results are presented and discussed.

Index Terms-Microstrip antennas, circular polarization, lowcost substrate, GPS.

\section{INTRODUÇÃO}

A polarização de uma antena, como estabelecido em [1], é definida pelo estado de polarização da onda eletromagnética por ela irradiada. Dessa forma, quando se afirma que uma antena é circularmente polarizada (CP), está implícito nesta afirmação que o campo elétrico distante por ela irradiado possui duas componentes, uma na direção $\theta$ e outra na direção $\phi$, relativas a um sistema de coordenadas esféricas, de mesma intensidade, porém defasadas, no tempo, de $90^{\circ}$. Antenas de microfita, classificadas como irradiadores do tipo ressonante, podem satisfazer estes requisitos. Das diversas topologias desenvolvidas para gerar ondas CP [2], a quadrada de canto truncado e a quase-quadrada, ambas alimentadas por uma ponta de prova coaxial, têm sido utilizadas como antenas de recepção de sinais de GPS [3-4].

O procedimento de projeto destas antenas encontra-se bem estabelecido na literatura [5-6]. Seguindo-o, antena com excelente razão axial na freqüência central da faixa de operação do GPS pode ser obtida. Entretanto, sua impedância de entrada é inerentemente indutiva, principalmente se substratos espessos forem utilizados com o objetivo de aumentar sua faixa de passagem de razão axial (RA). Uma forma capaz de compensar este efeito é a utilização de um capacitor anular ou com contorno fractal construído no próprio elemento, concêntrico ou não à ponta de prova coaxial de alimentação, como reportado em [3-4].

Neste trabalho, ao invés de se utilizar desta técnica, um novo procedimento é proposto para o projeto de antenas com cantos truncados alimentadas por um conector SMA, com resultados muito bons, mesmo no caso de antenas espessas.

D.C. Nascimento, R. Schildberg e J.C.S. Lacava, Laboratório de Antenas e Propagação - LAP, Instituto Tecnológico de Aeronáutica, São José dos Campos, SP, E-mails: danielcnascimento@gmail.com, \{schild, lacava\}@ita.br. L. Cividanes, Divisão de Eletrônica Aeroespacial, INPE, São José dos Campos, SP, E-mail: lucio@dea.inpe.br. Este trabalho foi parcialmente financiado pela FINEP, projetos RHODES e CAPTAER.
Este procedimento incorpora a energia armazenada nos campos de franja do elemento, eminentemente capacitiva, ao projeto da antena, visando bloquear o efeito indutivo do condutor central do conector SMA de alimentação. Dessa forma, é possível posicionar a freqüência de melhor razão axial próxima a de melhor casamento de impedância, sem o auxílio de estruturas externas.

\section{ANTENA QUADRADA COM CANTOS TRUNCADOS}

A geometria típica da antena de microfita quadrada com cantos truncados (AMQCT) é mostrada na Fig. 1. Em geral, irradiadores AMQCT comerciais, objetivando reduzir suas dimensões, são projetados com laminados de microondas de baixas perdas como o Duroid TMM10 $\left(\varepsilon_{r}=9,9\right.$, espessura $=3,81 \mathrm{~mm}$ e tangente de perdas $=0,0027)$, muito caro para os padrões nacionais.

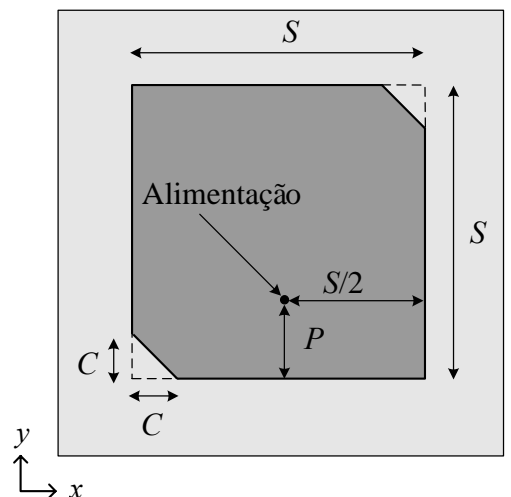

Fig. 1. Geometria típica da antena quadrada com cantos truncados.

Para entender melhor os problemas relacionados com este tipo de antena, uma AMQCT, com plano de terra e dielétrico finitos $\left(50 \times 50 \mathrm{~mm}^{2}\right)$, circularmente polarizada para a direita, foi projetada segundo o procedimento padrão descrito na literatura, para operar na freqüência L1 do GPS. Tal projeto foi realizado com auxílio do programa HFSS, resultando na antena com dimensões $S=28,42 \mathrm{~mm}, C=2,6 \mathrm{~mm}$ e $P=9,71 \mathrm{~mm}$. Simulações revelaram que esta antena possui $4,5 d B$ de diretividade, faixa de passagem para RA de $3 d B$ igual a 9,9\% e $82 \%$ de eficiência de irradiação. Sua impedância de entrada, traçada sobre a carta de Smith, é mostrado na Fig. 2. Nota-se deste gráfico que na freqüência de projeto, a impedância de entrada é ligeiramente indutiva, com $Z_{\text {in }}=50,7+\mathrm{j} 7,9 \Omega$, de modo que a antena está relativamente bem casada com o conector SMA de $50 \Omega$ de impedância característica. Claramente, estes parâmetros satisfazem as especificações do sistema GPS [7].

Entretanto, se o mesmo procedimento for aplicado no projeto de uma antena de baixo custo, tendo, por exemplo, 
o FR4 $\left(\varepsilon_{r}=4,465\right.$ e tangente de perdas $\left.=0,03\right)$ como substrato, e se a mesma espessura 3,81 $\mathrm{mm}$ for utilizada, sua eficiência de irradiação é reduzida drasticamente, o que é inaceitável para a aplicação desejada. Uma forma de melhorar este parâmetro é a utilização de substrato espesso [4].

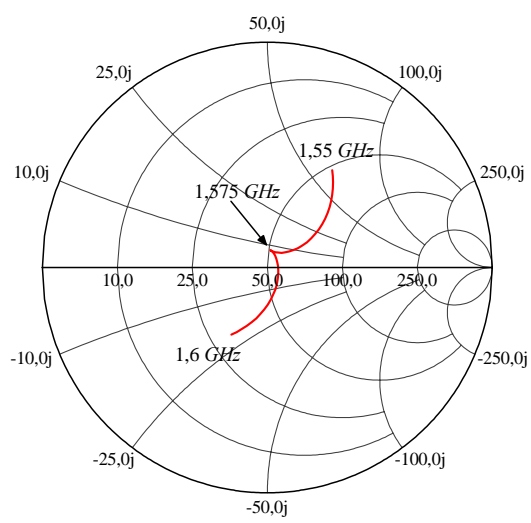

Fig. 2. Impedância de entrada da antena AMQCT com Duroid TMM10.

Empregando como substrato o FR4 com 6,2 $\mathrm{mm}$ de espessura, uma antena do tipo AMQCT, com plano de terra finito $\left(70 \times 70 \mathrm{~mm}^{2}\right)$, foi projetada para operar na freqüência L1 do GPS, de acordo com o procedimento padrão descrito na literatura. As seguintes dimensões foram obtidas após otimizações no HFSS: $S=41,9 \mathrm{~mm}, C=9,4 \mathrm{~mm}$ e $P=9,95 \mathrm{~mm}$. Os resultados para a razão axial, perda de retorno e impedância de entrada são apresentados nas Figs. 3 e 4.

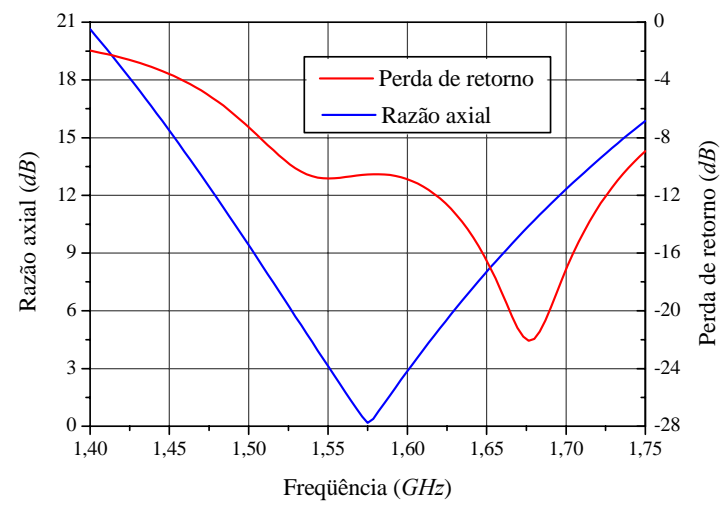

Fig. 3. Curvas para a perda de retorno e razão axial da antena AMQCT com FR4.

Nota-se da Fig. 4 que a impedância de entrada da antena AMQCT com substrato FR4, na freqüência L1, tem reatância elevada $\left(Z_{\text {in }}=48,4+\mathrm{j} 30,5 \Omega\right)$, de modo que esta não se encontra devidamente casada com o conector SMA de $50 \Omega$ de impedância característica. Além disso, a freqüência na qual a melhor razão axial ocorre $(1,575 \mathrm{GHz})$ não coincide com a de melhor casamento $(1,6765 \mathrm{GHz})$. A faixa de passagem de $3 d B$ para a razão axial, centrada em L1, é simétrica e corresponde a $50 \mathrm{MHz}$ (cerca de 3,2\%). Nesta faixa, a perda de retorno é estável, porém da ordem de $-10,6 d B$. Como esperado, devido à espessura utilizada, sua eficiência de irradiação é de 67 \%. Claramente alguns parâmetros satisfazem os requisitos do sistema GPS, mas a perda de retorno obtida, na faixa de melhor razão axial, não é aceitável. Este problema pode ser resolvido com auxílio de um capacitor fractal [3]. Neste trabalho, porém, um procedimento diferente é utilizado.

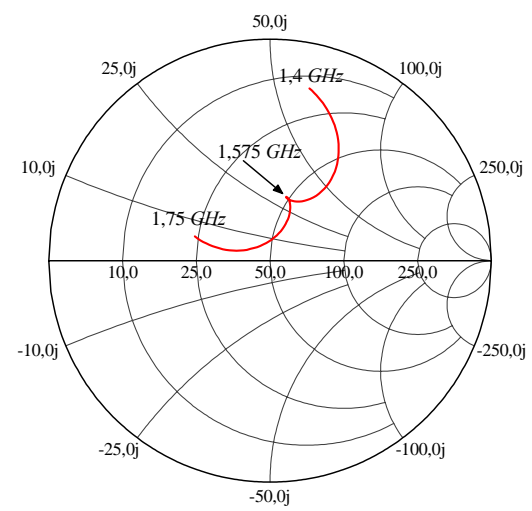

Fig. 4. Impedância de entrada da antena AMQCT com FR4.

\section{ANTENA RETANGULAR COM CANTOS TRUnCADOS}

Como mencionado em [3], o uso do FR4 como substrato introduz, devido à tolerância de sua permissividade relativa não ser especificada pelo fabricante, uma complexidade adicional ao projeto. Esse problema pode ser contornado com a adição de quatro tocos de sintonia ao elemento irradiador, como ilustrado na Fig. 5. Cortando adequadamente os tocos, o deslocamento da freqüência de operação, em função da variação da permissividade relativa, pode ser compensado. É importante salientar que em [3] o projeto foi realizado com o elemento irradiador quadrado ( $L_{1}$ igual a $\left.L_{2}\right)$.

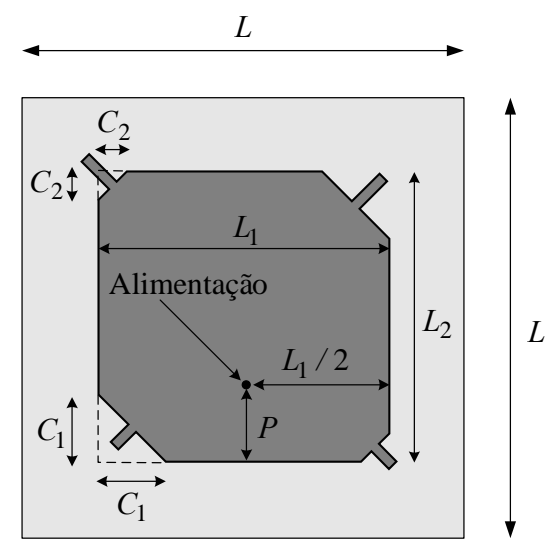

Fig. 5. Geometria típica da antena retangular com cantos truncados.

No novo procedimento de projeto, ao invés de quadrado, utiliza-se de um elemento retangular. Esta nova topologia é designada por AMRCT. Com os novos parâmetros de projeto, lados $L_{1}$ e $L_{2}$ diferentes, o desenvolvimento da antena é mais flexível. Empregando como substrato o mesmo FR4 com 6,2 $\mathrm{mm}$ de espessura, uma AMRCT com plano de terra finito $\left(70 \times 70 \mathrm{~mm}^{2}\right)$ foi projetada para operar em 1,57542 $\mathrm{GHz}$. Após otimizações no HFSS, foram obtidas as seguintes dimensões para a antena em questão: $L_{1}=41,00 \mathrm{~mm}, L_{2}=43,35 \mathrm{~mm}$, $C_{1}=10,00 \mathrm{~mm}, P=7,675 \mathrm{~mm} C_{2}=4,00 \mathrm{~mm}$, tocos maiores com comprimentos iguais a $5,50 \mathrm{~mm}$ e os menores com $3,50 \mathrm{~mm}$. Simulações mostraram que a AMRCT possui $5,86 d B$ de diretividade, 48,5 $\mathrm{MHz}$ (cerca de 3,08\%) de faixa de pas- 
sagem de $3 d B$ para a razão axial e $67 \%$ de eficiência de irradiação.

Na Fig. 6 são apresentadas as curvas para a razão axial e perda de retorno em função da freqüência. Sua impedância de entrada, medida com o analisador de redes vetorial HP 8714ET, é mostrada na Fig. 7, sobre a carta de Smith, juntamente com a expectativa teórica obtida com o HFSS. Gráficos para as partes real e imaginária de $Z_{\text {in }}$ são apresentados na Fig. 8. Das Figs. 7 e 8 é possível notar a excelente concordância entre os resultados teóricos e os experimentais obtidos para $Z_{i n}$.

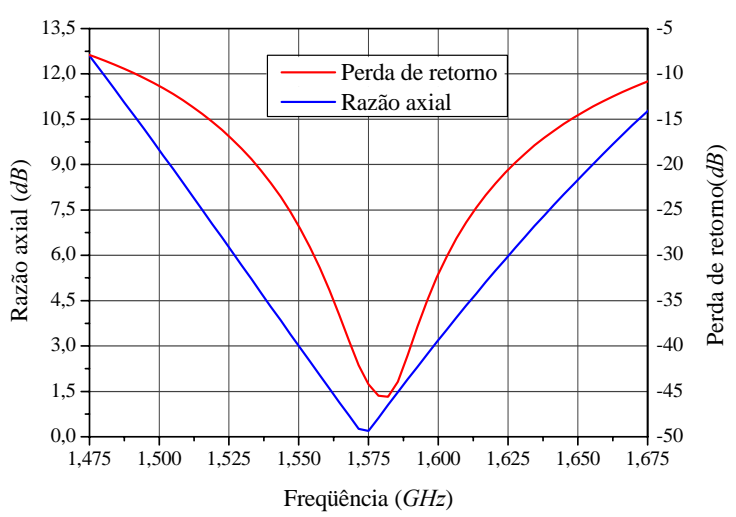

Fig. 6. Curvas para a perda de retorno e razão axial da antena AMRCT.

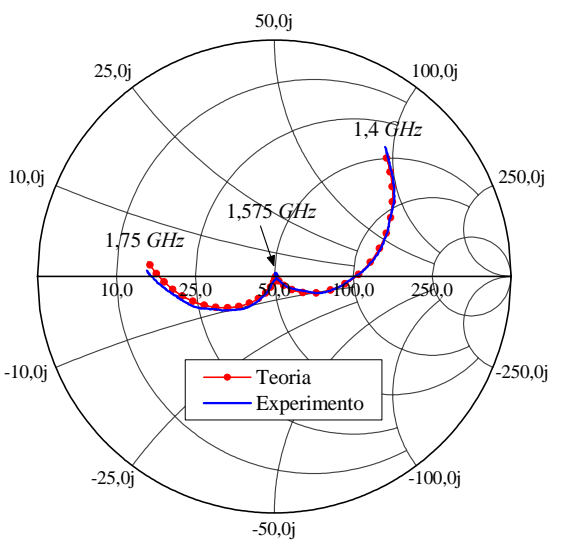

Fig. 7. Impedância de entrada da antena AMRCT.

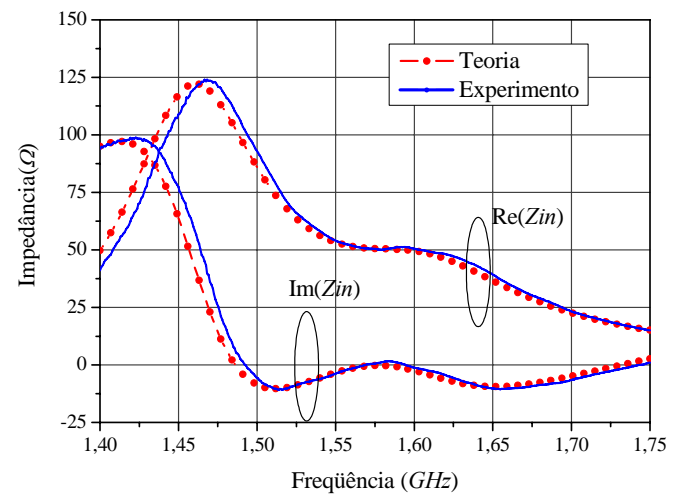

Fig. 8. Curvas para as partes real e imaginária de $Z_{\text {in }}$ da antena AMRCT.

Comparando os gráficos da Figs. 3 e 6 nota-se claramente que a nova abordagem de projeto realmente conseguiu posicionar a faixa de melhor casamento de impedância dentro da faixa de passagem da razão axial. Em particular, para a antena em questão, obteve-se $0,19 d B$ para a razão axial e $-45 d B$ para a perda de retorno, na freqüência L1 do GPS. Estes resultados, do ponto de vista de engenharia, são excelentes e comprovam não ser necessário, pelo menos nas condições em que o projeto foi realizado, se utilizar de técnicas complexas para posicionar a faixa de passagem de impedância próxima da de razão axial.

Diagramas de irradiação no plano $x z$, teóricos e experimentais, são apresentados nas Figs. 9 e 10 para as componentes $\theta$ e $\phi$ do campo elétrico irradiado, traçados na freqüência de 1,57542 GHz. Nas Figs. 11 e 12 são mostrados os resultados para o plano $y z$. Nota-se uma boa concordância entre teoria e experimento nos dois planos considerados.

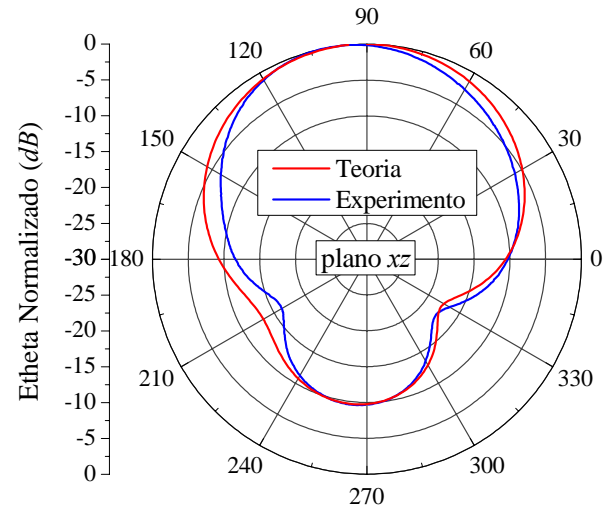

Fig. 9. Diagramas de irradiação da componente $\theta$ do campo elétrico irradiado, no plano $x z$, para a freqüência de $1,57542 \mathrm{GHz}$.

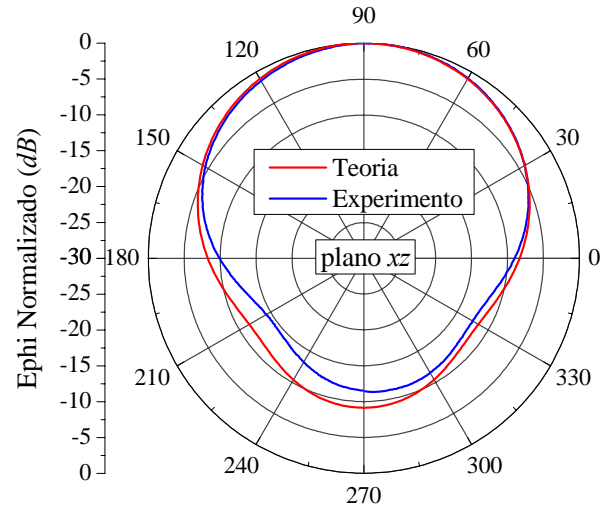

Fig. 10. Diagramas de irradiação da componente $\phi$ do campo elétrico irradiado, no plano $x z$, para a freqüência de $1,57542 \mathrm{GHz}$.

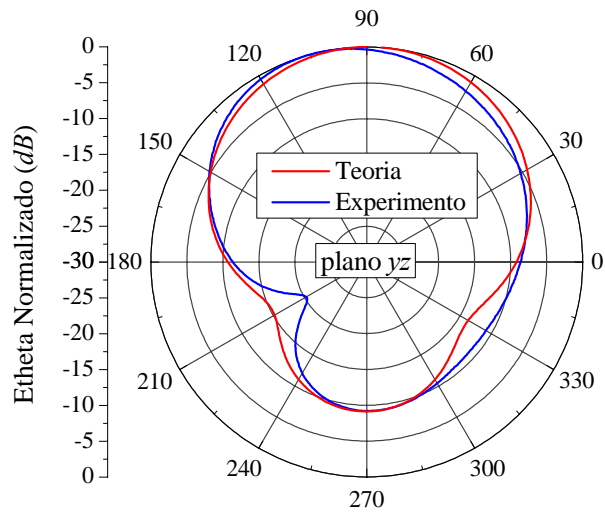

Fig. 11. Diagramas de irradiação da componente $\theta$ do campo elétrico irradiado, no plano $y z$, para a freqüência de $1,57542 \mathrm{GHz}$. 


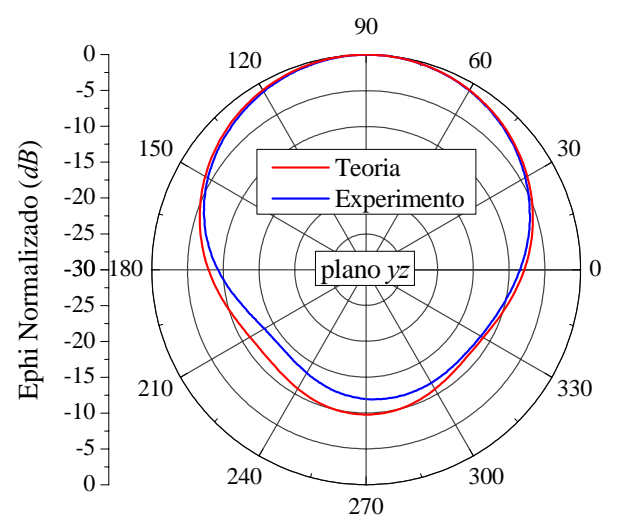

Fig. 12. Diagramas de irradiação da componente $\phi$ do campo elétrico irradiado, no plano $y z$, para a freqüência de $1,57542 \mathrm{GHz}$.

\section{SimULAÇÃO FINAL}

Até o presente estágio do projeto, somente o elemento irradiador foi enfocado. Entretanto, para torná-lo operacional, é necessário incorporar à simulação a base metálica que o sustentará e alojará seu LNA (amplificador de baixo ruído), além de suportar a cobertura (radome) de proteção ambiental. Como dielétrico de cobertura é utilizado o ABS, um termoplástico com 3,4 de permissividade relativa e, por ser um material de baixo custo, tem 0,07 de tangente de perdas.

Na Fig. 13 é mostrada a visão superior, sem a radome, da parte interna da estrutura na qual a antena se insere, apoiando-se sobre uma base metálica de $54,5 \mathrm{~mm}$ de raio e centrada nesta. A dimensão $G=1,5 \mathrm{~mm}$ nesta figura corresponde à espessura da parede lateral da radome de proteção da antena.

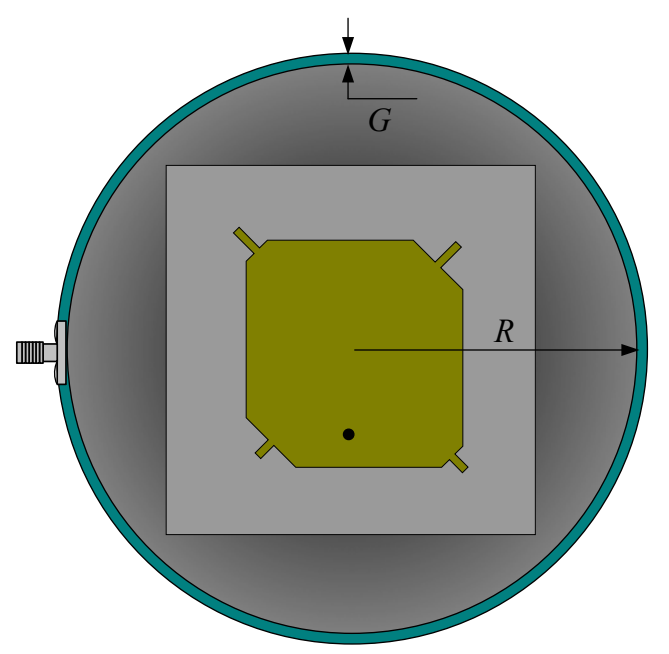

Fig. 13. Vista superior da estrutura metálica de sustentação da antena projetada.

Na Fig. 13 é mostrado um corte lateral da estrutura proposta. As dimensões cotadas nesta figura são: $H_{D}=6,2 \mathrm{~mm}$, que indica a espessura do substrato da antena, $T=1,5 \mathrm{~mm}$, que corresponde à penetração da mesma na base metálica de sustentação, que tem $H_{M}=13 \mathrm{~mm}$ de espessura. Visando minimizar o efeito da cobertura dielétrica sobre as características elétricas da antena, esta é posicionada a $3 \mathrm{~mm}$ distante da face superior do irradiador. Com estas dimensões é obtida uma estrutura com $H=22,2 \mathrm{~mm}$ de altura.

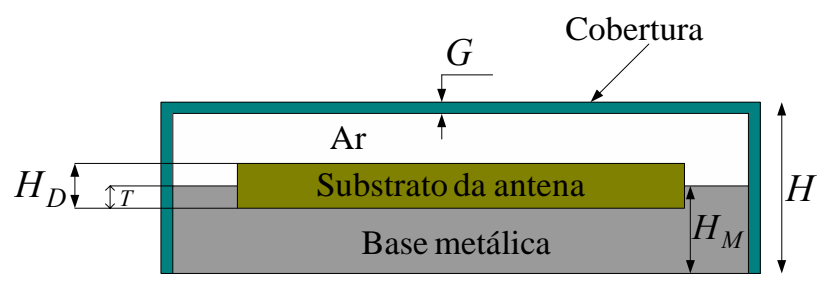

Fig. 14. Corte lateral da estrutura proposta para alojar a antena e seu LNA.

Com as dimensões da antena estabelecidas no item III, a estrutura mostrada nas Figs. 13 e 14 foi montada no ambiente do HFSS. Otimizações foram então realizadas até que os efeitos da camada de cobertura e da base metálica fossem neutralizados, resultando nas novas dimensões para a antena (com plano de terra finito de $70 \times 70 \mathrm{~mm}^{2}$ ): $L_{1}=41,05 \mathrm{~mm}, L_{2}=43,1 \mathrm{~mm}$, $C_{1}=9,4 \mathrm{~mm}, C_{2}=4,00 \mathrm{~mm}, P=8,3 \mathrm{~mm}$, tocos maiores com comprimentos iguais a 5,50 $\mathrm{mm}$ e os menores com 3,50 $\mathrm{mm}$.

Na Fig. 15 é mostrado o resultado da simulação realizada para a impedância de entrada, traçado sobre a carta de Smith. Curvas para a perda de retorno e razão axial são apresentadas na Fig. 16. Dessas curvas é possível visualizar que a nova abordagem de projeto conseguiu, mais uma vez, estabelecer a região de melhor casamento de impedância próxima da de melhor razão axial, validando a geometria proposta para alojar a antena e seu LNA.

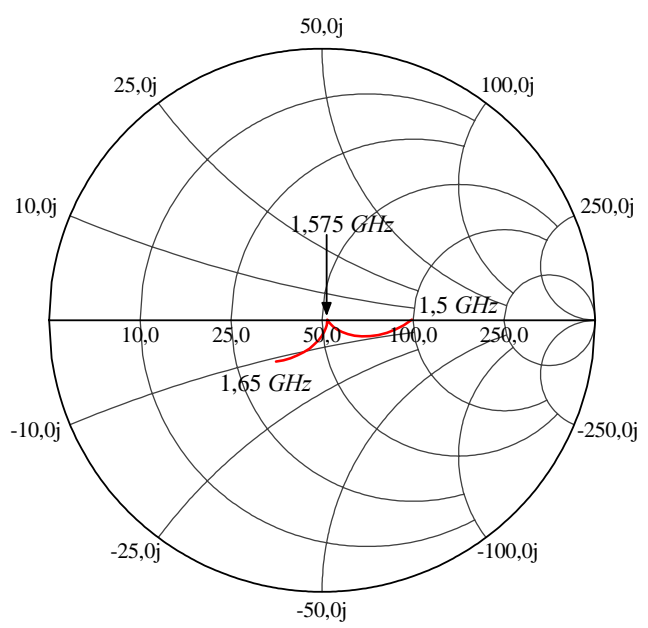

Fig. 15. Impedância de entrada da antena encapsulada.

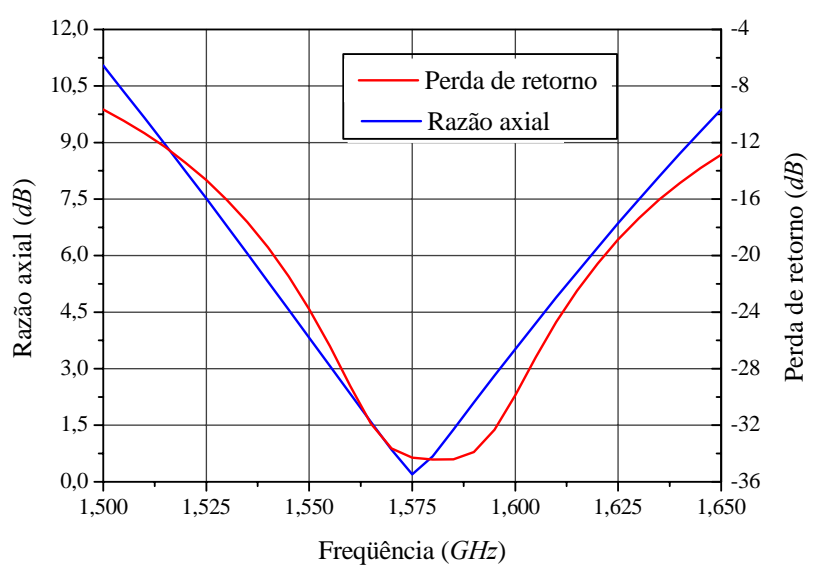

Fig. 16. Curvas para a perda de retorno e razão axial da antena encapsulada. 


\section{CONCLusÕES}

Neste trabalho foi apresentada uma nova metodologia para o projeto de antenas de microfita espessas com cantos truncados, alimentadas por pontas de provas coaxiais. Esta metodologia foi aplicada a uma antena de baixo custo, concebida para receber sinais do sistema GPS. Para atingir valores aceitáveis para a sua eficiência de irradiação, foi utilizado um substrato com 6,2 mm de espessura. Se uma antena tão espessa como esta tivesse sido projetada segundo o procedimento padrão descrito na literatura, sua impedância de entrada seria, certamente, fortemente indutiva. Para contornar este problema, ao invés da clássica geometria quadrada com cantos truncados, foi utilizada uma geometria retangular para o elemento irradiador. Esta mudança viabilizou o projeto de uma antena circularmente polarizada com excelentes resultados, tanto para a perda de retorno como para a razão axial, em toda a faixa de operação da freqüência L1 do GPS. Finalizando o trabalho, resultados de simulações para a impedância de entrada, perda de retorno e razão axial, com a antena posicionada na estrutura concebida para sua proteção ambiental e alojar seu LNA, foram apresentados.

\section{AGRADECIMENTOS}

Ao IFI (Instituto de Fomento e Coordenação Industrial) por permitir a utilização de sua câmara anecóica.

\section{REFERÊNCIAS}

[1] IEEE Standard Definitions of Terms for Antennas: IEEE Std 145-1983. Published by IEEE: New York, 1983.

[2] J.R. James and P.S. Hall (eds.), Handbook of microstrip antennas, vol. 1, London: Peter Peregrinus, 1989.

[3] D.C. Nascimento, J.A. Mores Jr., R. Schildberg e J.C.S. Lacava, "Low-cost truncated corner microstrip antenna for GPS application,” Proc. of the 2006 IEEE AP-S International Symposium, Albuquerque, NM, USA, pp. 1557 1560, July 2006.

[4] D.C. Nascimento, J.A. Mores Jr., R. Schildberg e J.C.S. Lacava, “Análise e projeto de antenas de microfita de baixo custo,” MOMAG 2006, CD-ROM, Belo Horizonte, MG, agosto de 2006.

[5] R. Garg, P. Bhartia, I. Bahl, and A. Ittipiboon, Microstrip antenna design handbook, Artech House, Norwood, 2001.

[6] J.R. James and P.S. Hall (eds.), Handbook of microstrip antennas, vol 1, Peter Peregrinus: London, 1989.

[7] B.W. Parkinson, J.J. Spilker Jr. Global positioning system: theory and applications, vol. 1, American Institute of Aeronautics and Astronautics, 1996. 